\title{
Stability analysis of nonlinear observer for neutral uncertain time-delay systems
}

\author{
Yali Dong ${ }^{1 *}$, Tianrui Li ${ }^{1}$ and Xuehua Zhang ${ }^{2}$
}

\section{"Correspondence:}

dongyl@vip.sina.com

'School of Science, Tianjin

Polytechnic University, Tianjin,

300387, China

Full list of author information is

available at the end of the article

\section{Springer}

\begin{abstract}
This note is concerned with the observer design problem for a class of nonlinear neutral systems with time delay. The problem addressed is to design a full-order observer that guarantees the asymptotic stability of an error dynamic system. Firstly, some sufficient conditions for the existence of observers of a class of nonlinear neutral systems with time-varying delay are presented. An effective algebraic matrix equation approach is developed. Then we give a design method of the observer that is dependent on the solution of a linear matrix inequality. Furthermore, robust observer designs for a class of nonlinear neutral systems with time delay and uncertainties are obtained. Finally, an example is given to show the effectiveness of our proposed approaches.
\end{abstract}

MSC: 93D20; 93E10; 93C10; 34D20

Keywords: nonlinear neutral systems; nonlinear observers; asymptotic stability; time-delay system; algebraic matrix equation

\section{Introduction}

The state estimation (or observer) problem has been widely developed throughout the past three decades. It is well known that all state variables are rarely available for direct online measurement in most cases. There is a substantial need for the reliable estimation of state variables, especially when they are used in the synthesis of model-based controllers or for process-monitoring purposes (see e.g. [1, 2]). Various methods such as algebraic, geometric, inversion approaches, generalized inverse, singular-value decomposition, and the Kronecker canonical form techniques have been used in the observer design. Also, different types of state observers such as reduced and minimal-order, full-order, unknown input, functional, disturbance decoupled, etc., have been studied. The observer technique has shown its successful applications not only in system monitoring and regulation but also in detecting as well as identifying failures in dynamical systems (see e.g. [3]). Furthermore, since the system uncertainties and exogenous disturbance input are unavoidable in modeling, the robust state observer design problem has been studied for many years in order to preserve the satisfactory observer action under system uncertainties and exogenous disturbances (see e.g. [4-6]).

It is also well known that the existence of time delay in a system may cause instability or bad system performance in closed feedback systems. The time delay phenomenon may be encountered in many practical systems such as the AIDS epidemic, aircraft stabilization, chemical engineering systems, inferred grinding model, manual control, neural 
network, nuclear reactor, population dynamic model, rolling mill, ship stabilization, and systems with lossless transmission lines. Hence stability analysis and observer design for time-delay systems have been investigated in recent years [7-11]. In the context of discrete delay systems, Trinh and Aldeen [12] proposed a memoryless state observer by the state augmentation approach; for continuous delay systems, a general form of linear observers was given in [13] by using the factorization approach, and a necessary and sufficient condition for the existence of state functional observers for such systems was presented. In many practical systems, the system models can be described by functional differential equations of neutral type, the models of which depend on both state and state derivatives. Neutral system examples include distributed networks, heat exchanges, and processes involving steam. Sufficient conditions have been proposed to guarantee the stability for neutral systems (see e.g. [14-18]).

On the other hand, the observer design problem for nonlinear systems has received considerable attention in the past years. By the coordinate transformation approach, a new constant gain observer design methodology for a class of multi-output nonlinear systems was proposed in [19], while in [20] a set of tools to design observers for nonlinear systems was developed. Recently, the observer design problem for the class of Lipschitz nonlinear systems without parameter uncertainty was addressed in [21, 22] respectively. An algebraic Riccati equation approach was adopted in [21]. In [23], the problem of observer design for a class of nonlinear discrete-time systems with time delay was considered. A new approach of nonlinear observer design was proposed for the class of systems. In [24], via state transformation and the constructive use of a Lyapunov function, the new observer design approach was addressed by introducing a parameter in the observer. Some sufficient conditions which guarantee the estimation error to asymptotically converge to zero under adaptive conditions were given. When parameter uncertainty as well as time delay appear simultaneously in the class of neutral nonlinear systems, it seems that little attention has been paid to the robust observer design problem so far.

In this paper, we address the observer design problem for nonlinear neutral systems with time-varying delay. Here, attention is focused on the design on a nonlinear observer such that the dynamics of the estimation error is asymptotically stable, dependent on the time delay. Some sufficient conditions are proposed to guarantee the existence of a desired observer. Furthermore, robust observer designs for a class of nonlinear neutral systems with time delay and uncertainties can be obtained. The method given in this note makes the applicable class larger than that given in the literature. A linear neutral system with constant time delay was considered in [25]. In this paper, we deal with the uncertain nonlinear neutral system with time-varying delay. Compared with [25], our results are applied in many more fields.

This paper is organized as follows. In Section 2, a problem formulation and preliminaries are stated. Observer design methodology for a class of neutral delay systems is presented in Section 3, and some sufficient conditions for the existence of the proposed observer are given. The observer design methodology for a class of uncertain nonlinear neutral delay systems is presented in Section 4. An illustrative example is given in Section 5. Concluding remarks are drawn in Section 6. 
2 Problem formulation and preliminaries

Consider nonlinear neutral delay-differential systems described by the following equation:

$$
\begin{aligned}
& \dot{x}(t)-J \dot{x}(t-d)=A x(t)+A_{1} x(t-h(t))+f(x(t), x(t-d), x(t-h(t))), \\
& x(t)=\varphi(t), \quad t \in[-\max \{d, v\}, 0], \\
& y(t)=C x(t)+C_{1} x(t-h(t)),
\end{aligned}
$$

where $x \in R^{n}$ is the state, $y \in R^{p}$ is the measurement output. $d$ denotes the constant time delay, $h(t)$ is a time-varying delay in the state satisfying

$$
0 \leq h(t) \leq v, \quad 0 \leq \dot{h}(t) \leq \lambda<1,
$$

where $v$ and $\lambda$ are constants. The spectrum radius of the matrix $J, \rho(J)$ satisfies $\rho(J)<1$. A, $A_{1}, J, C, C_{1}$ are known constant matrices with appropriate dimensions. $f: R^{n} \times R^{n} \times R^{n} \rightarrow$ $R^{n}$ is a continuous nonlinear function. $\varphi(t)$ is a continuous vector-valued initial function. In general, it is assumed that $f$ satisfies

$$
\begin{aligned}
& \|f(x(t), x(t-d), x(t-h(t)))-f(\hat{x}(t), \hat{x}(t-d), \hat{x}(t-h(t)))\|^{2} \\
& \quad \leq(x(t)-\hat{x}(t))^{T} Q_{1}(x(t)-\hat{x}(t))+(x(t-d)-\hat{x}(t-d))^{T} Q_{2}(x(t-d)-\hat{x}(t-d)) \\
& \quad+(x(t-h(t))-\hat{x}(t-h(t)))^{T} Q_{3}(x(t-h(t))-\hat{x}(t-h(t))), \\
& \quad \forall x(t), x(t-d), x(t-h(t)) \in R^{n},
\end{aligned}
$$

where $Q_{1}, Q_{2}$, and $Q_{3}$ are known positive definite matrices.

In this note, we consider the following full-order nonlinear observer:

$$
\begin{aligned}
\dot{\hat{x}}(t)-J \dot{\hat{x}}(t-d)= & A \hat{x}(t)+A_{1} \hat{x}(t-h(t))+f(\hat{x}(t), \hat{x}(t-d), \hat{x}(t-h(t))) \\
& +L\left[y(t)-C \hat{x}(t)-C_{1} \hat{x}(t-h(t))\right],
\end{aligned}
$$

where the constant matrix $L$ is the observer parameter vector.

Let the error state be

$$
e(t)=x(t)-\hat{x}(t)
$$

then it follows from (1)-(3) that

$$
\dot{e}(t)-J \dot{e}(t-d)=A_{c} e(t)+A_{d} e(t-h(t))+\Delta f
$$

where

$$
\begin{aligned}
& A_{c}=A-L C, \quad A_{d}=A_{1}-L C_{1}, \\
& \Delta f=f(x(t), x(t-d), x(t-h(t)))-f(\hat{x}(t), \hat{x}(t-d), \hat{x}(t-h(t))) .
\end{aligned}
$$

The following lemmas will be used in the development of the main results. 
Lemma 1 [21] Let $a \in R^{n}, b \in R^{n}$, and $\varepsilon>0$. Then we have

$$
a^{T} b+b^{T} a \leq \varepsilon a^{T} a+\varepsilon^{-1} b^{T} b .
$$

Lemma 2 [12] Given constant symmetric matrices $S_{1}, S_{2}, S_{3}$, and $S_{1}=S_{1}^{T}<0, S_{3}=S_{3}^{T}>0$, then $S_{1}+S_{2} S_{3}^{-1} S_{2}^{T}<0$ if and only if

$$
\left[\begin{array}{cc}
S_{1} & S_{2} \\
S_{2}^{T} & -S_{3}
\end{array}\right]<0 .
$$

Lemma 3 [14] Let $D, E$, and $F$ be real matrices of appropriate dimensions with $F^{T} F \leq I$, then for any scalar $\varepsilon>0$, we have the following inequality:

$$
D F E+E^{T} F^{T} D^{T} \leq \varepsilon^{-1} D D^{T}+\varepsilon E^{T} E .
$$

\section{Observer design for a class of neutral time-delay systems}

The next theorem will show that the asymptotic stability of system (5) is related to the existence of a positive definite solution to an algebraic matrix equation and, therefore, offers a key for solving the addressed observer design problem.

Theorem 1 For given sufficiently small scalars $\sigma_{1}, \sigma_{2}>0$ and matrices $L, S>0$, error system (5) is asymptotically stable if there exist positive scalars $\varepsilon_{i}(i=1,2, \ldots, 5)$ and a positive definite matrix $P$ satisfying the following matrix equation:

$$
\begin{array}{r}
P A_{c}+A_{c}^{T} P+\varepsilon_{1}^{-1} P^{2}+\varepsilon_{2} A_{c}^{T} A_{c}+\varepsilon_{4}^{-1} P^{2}+\left(\varepsilon_{4}+\varepsilon_{5}\right)\left(Q_{1}+Q_{2}+Q_{3} /(1-\lambda)\right)+\sigma_{1} I \\
+\left(\varepsilon_{2}^{-1}+\varepsilon_{3}^{-1}+\varepsilon_{5}^{-1}\right) J^{T} P^{2} J+\left(\left(\varepsilon_{1}+\varepsilon_{3}\right) /(1-\lambda)\right) A_{d}^{T} A_{d}+\left(\sigma_{2} /(1-\lambda)\right) I+S=0 .
\end{array}
$$

Proof Consider the following candidate Lyapunov-Krasovskii functional:

$$
\begin{aligned}
V(e(t), t)= & (e(t)-J e(t-d))^{T} P(e(t)-J e(t-d))+\int_{t-d}^{t} e^{T}(s) Q e(s) d s \\
& +\int_{t-h(t)}^{t} e^{T}(s) \operatorname{Re}(s) d s .
\end{aligned}
$$

The derivative of $V$ along a given trajectory is obtained as

$$
\begin{aligned}
\frac{d}{d t} V(e(t), t)= & 2(e(t)-J e(t-d))^{T} P\left(A_{c} e(t)+A_{d} e(t-h(t))+\Delta f\right)+e^{T}(t) Q e(t) \\
& -e^{T}(t-d) Q e(t-d)+e^{T}(t) \operatorname{Re}(t)-(1-\dot{h}(t)) e^{T}(t-h(t)) \operatorname{Re}(t-h(t)) \\
\leq & e^{T}(t)\left(P A_{c}+A_{c}^{T} P\right) e(t)+e^{T}(t) P A_{d} e(t-h(t))+e^{T}(t-h(t)) A_{d}^{T} \operatorname{Pe}(t) \\
& -e^{T}(t-d) J^{T} P A_{c} e(t)-e^{T}(t) A_{c}^{T} P J e(t-d)-e^{T}(t-d) J^{T} P A_{d} e(t-h(t)) \\
& -e^{T}(t-h(t)) A_{d}^{T} P J e(t-d)+e^{T}(t) P \Delta f+(\Delta f)^{T} \operatorname{Pe}(t) \\
& -e^{T}(t-d) J^{T} P \Delta f-(\Delta f)^{T} \operatorname{PJe}(t-d)+e^{T}(t) \operatorname{Qe}(t) \\
& -e^{T}(t-d) Q e(t-d)+e^{T}(t) \operatorname{Re}(t)-(1-\lambda) e^{T}(t-h(t)) \operatorname{Re}(t-h(t)) .
\end{aligned}
$$


From Lemma 1, we obtain

$$
\begin{aligned}
& e^{T}(t) P A_{d} e(t-h(t))+e^{T}(t-h(t)) A_{d}^{T} P e(t) \\
& \quad \leq \varepsilon_{1}^{-1} e^{T}(t) P^{2} e(t)+\varepsilon_{1} e^{T}(t-h(t)) A_{d}^{T} A_{d} e(t-h(t)), \\
& -e^{T}(t-d) J^{T} P A_{c} e(t)-e^{T}(t) A_{c}^{T} P J e(t-d) \\
& \quad \leq \varepsilon_{2} e^{T}(t) A_{c}^{T} A_{c} e(t)+\varepsilon_{2}^{-1} e^{T}(t-d) J^{T} P^{2} J e(t-d), \\
& -e^{T}(t-d) J^{T} P A_{d} e(t-h(t))-e^{T}(t-h(t)) A_{d}^{T} P J e(t-d) \\
& \quad \leq \varepsilon_{3} e^{T}(t-h(t)) A_{d}^{T} A_{d} e(t-h(t))+\varepsilon_{3}^{-1} e^{T}(t-d) J^{T} P^{2} J e(t-d), \\
& e^{T}(t) P \Delta f+(\Delta f)^{T} P e(t) \leq \varepsilon_{4}^{-1} e^{T}(t) P^{2} e(t)+\varepsilon_{4}\|\Delta f\|^{2}, \\
& -e^{T}(t-d) J^{T} P \Delta f-(\Delta f)^{T} P J e(t-d) \leq \varepsilon_{5}^{-1} e^{T}(t-d) J^{T} P^{2} J e(t-d)+\varepsilon_{5}\|\Delta f\|^{2} .
\end{aligned}
$$

Substituting (10)-(14) into (9), we have

$$
\begin{aligned}
\frac{d}{d t} V(e(t), t) \leq & e^{T}(t)\left(P A_{c}+A_{c}^{T} P\right) e(t)+\varepsilon_{1}^{-1} e^{T}(t) P^{2} e(t)+\varepsilon_{1} e^{T}(t-h(t)) A_{d}^{T} A_{d} e(t-h(t)) \\
& +\varepsilon_{2} e^{T}(t) A_{c}^{T} A_{c} e(t)+\varepsilon_{2}^{-1} e^{T}(t-d) J^{T} P^{2} J e(t-d) \\
& +\varepsilon_{3} e^{T}(t-h(t)) A_{d}^{T} A_{d} e(t-h(t))+\varepsilon_{3}^{-1} e^{T}(t-d) J^{T} P^{2} J e(t-d) \\
& +\varepsilon_{4}^{-1} e^{T}(t) P^{2} e(t)+\varepsilon_{4}\|\Delta f\|^{2}+\varepsilon_{5}^{-1} e^{T}(t-d) J^{T} P^{2} J e(t-d)+\varepsilon_{5}\|\Delta f\|^{2} \\
& +e^{T}(t) Q e(t)-e^{T}(t-d) Q e(t-d) \\
& +e^{T}(t) \operatorname{Re}(t)-(1-\lambda) e^{T}(t-h(t)) \operatorname{Re}(t-h(t)) \\
= & e^{T}(t)\left(P A_{c}+A_{c}^{T} P+\varepsilon_{1}^{-1} P^{2}+\varepsilon_{2} A_{c}^{T} A_{c}+\varepsilon_{4}^{-1} P^{2}+Q+R\right) e(t) \\
& +e^{T}(t-h(t))\left(\varepsilon_{1} A_{d}^{T} A_{d}+\varepsilon_{3} A_{d}^{T} A_{d}-(1-\lambda) R\right) e(t-h(t)) \\
& +e^{T}(t-d)\left(\varepsilon_{2}^{-1} J^{T} P^{2} J+\varepsilon_{3}^{-1} J^{T} P^{2} J\right. \\
& \left.+\varepsilon_{5}^{-1} J^{T} P^{2} J-Q\right) e(t-d)+\left(\varepsilon_{4}+\varepsilon_{5}\right)\|\Delta f\|^{2} \\
\leq & e^{T}(t)\left(P A_{c}+A_{c}^{T} P+\varepsilon_{1}^{-1} P^{2}+\varepsilon_{2} A_{c}^{T} A_{c}+\varepsilon_{4}^{-1} P^{2}+Q+R+\left(\varepsilon_{4}+\varepsilon_{5}\right) Q_{1}\right) e(t) \\
& +e^{T}(t-h(t))\left(\varepsilon_{1} A_{d}^{T} A_{d}+\varepsilon_{3} A_{d}^{T} A_{d}-(1-\lambda) R+\left(\varepsilon_{4}+\varepsilon_{5}\right) Q_{3}\right) e(t-h(t)) \\
& +e^{T}(t-d)\left(\varepsilon_{2}^{-1} J^{T} P^{2} J+\varepsilon_{3}^{-1} J^{T} P^{2} J+\varepsilon_{5}^{-1} J^{T} P^{2} J-Q+\left(\varepsilon_{4}+\varepsilon_{5}\right) Q_{2}\right) e(t-d) .
\end{aligned}
$$

Let

$$
\begin{aligned}
& Q:=\varepsilon_{2}^{-1} J^{T} P^{2} J+\varepsilon_{3}^{-1} J^{T} P^{2} J+\varepsilon_{5}^{-1} J^{T} P^{2} J+\left(\varepsilon_{4}+\varepsilon_{5}\right) Q_{2}+\sigma_{1} I, \\
& (1-\lambda) R=\varepsilon_{1} A_{d}^{T} A_{d}+\varepsilon_{3} A_{d}^{T} A_{d}+\left(\varepsilon_{4}+\varepsilon_{5}\right) Q_{3}+\sigma_{2} I .
\end{aligned}
$$

Then we get

$$
\begin{aligned}
\frac{d}{d t} V(e(t), t) \leq & e^{T}(t)\left(P A_{c}+A_{c}^{T} P+\varepsilon_{1}^{-1} P^{2}+\varepsilon_{2} A_{c}^{T} A_{c}+\varepsilon_{4}^{-1} P^{2}+Q+R+\left(\varepsilon_{4}+\varepsilon_{5}\right) Q_{1}\right) e(t) \\
& -\sigma_{2} e^{T}(t-h(t)) e(t-h(t))-\sigma_{1} e^{T}(t-d) e(t-d)
\end{aligned}
$$


For simplicity, we denote

$$
\Pi:=P A_{c}+A_{c}^{T} P+\varepsilon_{1}^{-1} P^{2}+\varepsilon_{2} A_{c}^{T} A_{c}+\varepsilon_{4}^{-1} P^{2}+Q+R+\left(\varepsilon_{4}+\varepsilon_{5}\right) Q_{1},
$$

where $Q$ and $R$ are given by (15) and (16).

From (7), (15), and (16), we get that $\Pi=-S<0$.

Substituting (18) into (17) yields

$$
\begin{aligned}
\frac{d}{d t} V(e(t), t) & \leq e^{T}(t) \Pi e(t)-\sigma_{1} e^{T}(t-h(t)) e(t-h(t))-\sigma_{2} e^{T}(t-d) e(t-d) \\
& =\left[\begin{array}{c}
e(t) \\
e(t-h(t) \\
e(t-d)
\end{array}\right]\left[\begin{array}{ccc}
\Pi & 0 & 0 \\
0 & -\sigma_{1} I & 0 \\
0 & 0 & -\sigma_{2} I
\end{array}\right]\left[\begin{array}{c}
e(t) \\
e(t-h(t) \\
e(t-d)
\end{array}\right] \\
& \leq-\min \left(\lambda_{\min }(-\Pi), \sigma_{1}, \sigma_{2}\right)\left\|\left[\begin{array}{c}
e(t) \\
e(t-h(t) \\
e(t-d)
\end{array}\right]\right\|^{2} \\
& \leq-\min \left(\lambda_{\min }(-\Pi), \sigma_{1}, \sigma_{2}\right)\|e(t)\|^{2}<0,
\end{aligned}
$$

which implies that system (5) is asymptotically stable. This completes the proof of Theorem 1.

Remark 1 The use of the matrix $S>0$ is just to ensure that $\Pi<0$. In general, the positivedefinite matrix should be chosen sufficiently small in a matrix norm sense.

Theorem 2 For the given matrix $L$, error system (5) is asymptotically stable if there exist a positive definite matrix $P>0$ and positive scalars $\varepsilon_{i}(i=1,2, \ldots, 5)$ satisfying the following LMI:

$$
\left(\begin{array}{cccccc}
\Pi_{1} & P & P & J^{T} P & J^{T} P & J^{T} P \\
P & -\varepsilon_{1} I & 0 & 0 & 0 & 0 \\
P & 0 & -\varepsilon_{4} I & 0 & 0 & 0 \\
P J & 0 & 0 & -\varepsilon_{2} I & 0 & 0 \\
P J & 0 & 0 & 0 & -\varepsilon_{3} I & 0 \\
P J & 0 & 0 & 0 & 0 & -\varepsilon_{5} I
\end{array}\right),
$$

where

$$
\Pi_{1}=P A_{c}+A_{c}^{T} P+\varepsilon_{2} A_{c}^{T} A_{c}+\left(\varepsilon_{4}+\varepsilon_{5}\right)\left(Q_{1}+Q_{2}+\frac{1}{1-\lambda} Q_{3}\right)+\frac{\varepsilon_{1}+\varepsilon_{3}}{1-\lambda} A_{d}^{T} A_{d} .
$$

Proof Consider the following candidate Lyapunov-Krasovskii functional:

$$
\begin{aligned}
V(e(t), t)= & (e(t)-J e(t-d))^{T} P(e(t)-J e(t-d))+\int_{t-d}^{t} e^{T}(s) Q e(s) d s \\
& +\int_{t-h(t)}^{t} e^{T}(s) \operatorname{Re}(s) d s .
\end{aligned}
$$


Similar to the proof of Theorem 1, we have

$$
\begin{aligned}
\frac{d}{d t} V(e(t), t) \leq & e^{T}(t)\left(P A_{c}+A_{c}^{T} P+\varepsilon_{1}^{-1} P^{2}+\varepsilon_{2} A_{c}^{T} A_{c}+\varepsilon_{4}^{-1} P^{2}+Q+R+\left(\varepsilon_{4}+\varepsilon_{5}\right) Q_{1}\right) e(t) \\
& +e^{T}(t-h(t))\left(\varepsilon_{1} A_{d}^{T} A_{d}+\varepsilon_{3} A_{d}^{T} A_{d}-(1-\lambda) R+\left(\varepsilon_{4}+\varepsilon_{5}\right) Q_{3}\right) e(t-h(t)) \\
& +e^{T}(t-d)\left(\varepsilon_{2}^{-1} J^{T} P^{2} J+\varepsilon_{3}^{-1} J^{T} P^{2} J+\varepsilon_{5}^{-1} J^{T} P^{2} J-Q+\left(\varepsilon_{4}+\varepsilon_{5}\right) Q_{2}\right) e(t-d) .
\end{aligned}
$$

Let

$$
\begin{aligned}
& Q=\varepsilon_{2}^{-1} J^{T} P^{2} J+\varepsilon_{3}^{-1} J^{T} P^{2} J+\varepsilon_{5}^{-1} J^{T} P^{2} J+\left(\varepsilon_{4}+\varepsilon_{5}\right) Q_{2}, \\
& (1-\lambda) R=\varepsilon_{1} A_{d}^{T} A_{d}+\varepsilon_{3} A_{d}^{T} A_{d}+\left(\varepsilon_{4}+\varepsilon_{5}\right) Q_{3} .
\end{aligned}
$$

Then

$$
\frac{d}{d t} V(e(t), t) \leq e^{T}(t) \Phi e(t)
$$

where $\Phi=P A_{c}+A_{c}^{T} P+\varepsilon_{1}^{-1} P^{2}+\varepsilon_{2} A_{c}^{T} A_{c}+\varepsilon_{4}^{-1} P^{2}+Q+R+\left(\varepsilon_{4}+\varepsilon_{5}\right) Q_{1}$.

From (19) and Lemma 2, we have $\Phi<0$, which implies that system (5) is asymptotically stable. This completes the proof of Theorem 2.

Consider the neutral function differential system described by the following state equation:

$$
\begin{aligned}
& \dot{x}(t)-J \dot{x}(t-d)=A x(t)+A_{1} x(t-d)+f(t, x(t), x(t-d)), \\
& y(t)=C x(t)+C_{1} x(t-d),
\end{aligned}
$$

where $x \in R^{n}$ is the state, $y \in R^{p}$ is the measurement output. $d$ denotes the constant time delay. $A, A_{1}, J, C, C_{1}$ are known constant matrices with appropriate dimensions. $f: R \times$ $R^{n} \times R^{n} \rightarrow R^{n}$ is a continuous nonlinear function, and there exist positive definite matrices $T_{1}$ and $T_{2}$ such that

$$
\begin{aligned}
& \|f(t, x(t), x(t-d))-f(t, \hat{x}(t), \hat{x}(t-d))\|^{2} \\
& \quad \leq(x(t)-\hat{x}(t))^{T} T_{1}(x(t)-\hat{x}(t))+(x(t-d)-\hat{x}(t-d))^{T} T_{2}(x(t-d)-\hat{x}(t-d)) .
\end{aligned}
$$

We consider the following full-order nonlinear observer:

$$
\begin{aligned}
\dot{\hat{x}}(t)-J \dot{\hat{x}}(t-d)= & A \hat{x}(t)+A_{1} \hat{x}(t-d)+f(t, \hat{x}(t), \hat{x}(t-d)) \\
& +L\left[y(t)-C \hat{x}(t)-C_{1} \hat{x}(t-d)\right],
\end{aligned}
$$

where the constant matrix $L$ is the observer parameter vector.

Let the error state be

$$
e(t)=x(t)-\hat{x}(t)
$$


Then it follows from (20)-(23) that

$$
\dot{e}(t)-J \dot{e}(t-d)=A_{c} e(t)+A_{d} e(t-d)+\Delta f
$$

where $A_{c}=A-L C, A_{d}=A_{1}-L C_{1}, \Delta f=f(t, x(t), x(t-d))-f(t, \hat{x}(t), \hat{x}(t-d))$.

Corollary 1 For given sufficiently small scalar $\sigma_{1}>0$ and matrices $L, S>0$, error system (24) is asymptotically stable if there exist positive scalars $\varepsilon_{i}(i=1,2, \ldots, 5)$ and a positive definite matrix $P$ satisfying the following matrix equation:

$$
\begin{aligned}
& P A_{c}+A_{c}^{T} P+\varepsilon_{1}^{-1} P^{2}+\varepsilon_{2} A_{c}^{T} A_{c}+\varepsilon_{4}^{-1} P^{2}+\left(\varepsilon_{4}+\varepsilon_{5}\right)\left(T_{1}+T_{2}\right)+\sigma_{1} I \\
& \quad+\varepsilon_{1} A_{d}^{T} A_{d}+\varepsilon_{2}^{-1} J^{T} P^{2} J+\varepsilon_{5}^{-1} J^{T} P^{2} J+\varepsilon_{3}^{-1} J^{T} P^{2} J+\varepsilon_{3} A_{d}^{T} A_{d}+S=0 .
\end{aligned}
$$

Proof Similar to the proof of Theorem 1, condition (25) in Corollary 1 can be obtained and the detailed proof is omitted.

Corollary 2 For the given matrix L, error system (24) is asymptotically stable if there exist a positive definite matrix $P>0$ and positive scalars $\varepsilon_{i}(i=1,2, \ldots, 5)$, satisfying the following LMI:

$$
\left(\begin{array}{cccccc}
\bar{\Pi}_{1} & P & P & J^{T} P & J^{T} P & J^{T} P \\
P & -\varepsilon_{1} I & 0 & 0 & 0 & 0 \\
P & 0 & -\varepsilon_{4} I & 0 & 0 & 0 \\
P J & 0 & 0 & -\varepsilon_{2} I & 0 & 0 \\
P J & 0 & 0 & 0 & -\varepsilon_{3} I & 0 \\
P J & 0 & 0 & 0 & 0 & -\varepsilon_{5} I
\end{array}\right)<0,
$$

where

$$
\bar{\Pi}_{1}=P A_{c}+A_{c}^{T} P+\varepsilon_{2} A_{c}^{T} A_{c}+\left(\varepsilon_{4}+\varepsilon_{5}\right)\left(T_{1}+T_{2}\right)+\left(\varepsilon_{1}+\varepsilon_{3}\right) A_{d}^{T} A_{d} .
$$

Proof Similar to the proof of Theorem 2, condition (26) in Corollary 2 can be obtained and the detailed proof is omitted.

\section{Observer design for an uncertain neutral function differential system}

Consider an uncertain neutral function differential system described by the following state equation:

$$
\begin{aligned}
& \dot{x}(t)-J \dot{x}(t-d)=(A+\Delta A(t)) x(t)+\left(A_{d}+\Delta A_{d}(t)\right) x(t-d)+f(t, x(t), x(t-d)), \\
& x(t)=\varphi(t), \quad t \in[-d, 0], \\
& y(t)=C x(t),
\end{aligned}
$$

where $x \in R^{n}$ is the state vector, $y \in R^{p}$ is the measurement output. $d$ denotes the constant time delay. The spectrum radius of the matrix $J, \rho(J)$ satisfies $\rho(J)<1 . A, A_{d}, J, C$ are known constant matrices with appropriate dimensions. $\varphi(t)$ is a continuous vector-valued initial 
function. $f: R \times R^{n} \times R^{n} \rightarrow R^{n}$ is a continuous nonlinear function satisfying $f(t, 0,0)=0$, and there exist positive definite matrices $T_{1}$ and $T_{2}$ such that

$$
\begin{aligned}
& \|f(t, x(t), x(t-d))-f(t, \hat{x}(t), \hat{x}(t-d))\|^{2} \\
& \quad \leq(x(t)-\hat{x}(t))^{T} T_{1}(x(t)-\hat{x}(t))+(x(t-d)-\hat{x}(t-d))^{T} T_{2}(x(t-d)-\hat{x}(t-d))
\end{aligned}
$$

for $t \in R, x(t), x(t-d) \in R^{n} . \Delta A(t), \Delta A_{d}(t)$, and $\Delta B(t)$ are time-varying uncertainties, which satisfy the following conditions:

$$
\Delta A(t)=D F(t) E, \quad \Delta A_{d}(t)=D_{d} F(t) E_{d}
$$

where $D, E, D_{d}, E_{d}$ are real constant matrices of appropriate dimensions, and $F(t)$ is an unknown time-varying matrix with $F^{T}(t) F(t) \leq I$.

In this note, we consider the following full-order nonlinear observer:

$$
\dot{\hat{x}}(t)-j \dot{\hat{x}}(t-d)=A \hat{x}(t)+A_{d} \hat{x}(t-d)+f(t, \hat{x}(t), \hat{x}(t-d))+L(y(t)-C \hat{x}(t)),
$$

where the constant matrix $L$ is the observer parameter vector.

Let the error state be

$$
e(t)=x(t)-\hat{x}(t)
$$

Then it follows from (27)-(30) that

$$
\dot{e}(t)-J \dot{e}(t-d)=A_{c} e(t)+\Delta A(t) x(t)+\Delta A_{d}(t) x(t-d)+A_{d} e(t-d)+\Delta f,
$$

where $A_{c}=A-L C, \Delta f=f(t, x(t), x(t-d))-f(t, \hat{x}(t), \hat{x}(t-d))$.

Consider the following nonlinear system:

$$
\begin{aligned}
& \dot{x}(t)-J \dot{x}(t-d)=(A+\Delta A(t)) x(t)+\left(A_{d}+\Delta A_{d}(t)\right) x(t-d)+f(t, x(t), x(t-d)), \\
& \dot{e}(t)-J \dot{e}(t-d)=A_{c} e(t)+\Delta A(t) x(t)+\Delta A_{d}(t) x(t-d)+A_{d} e(t-d)+\Delta f .
\end{aligned}
$$

In the following theorem, a sufficient condition is derived so as to guarantee the asymptotic stability for system (33).

Theorem 3 For given sufficiently small positive scalars $\delta_{1}, \delta_{2}$ and matrix $L$, and positive definite matrices $R_{1}, R_{2}$, system (33) is asymptotically stable if there exist positive scalars $\varepsilon_{i}(i=1,2, \ldots, 10)$ and positive definite matrices $P_{1}$ and $P_{2}$ satisfying the following matrix equations:

$$
\begin{aligned}
& P_{1} A_{c}+A_{c}^{T} P_{1}+P_{1} D D^{T} P_{1}+\left(\varepsilon_{1}^{-1}+\varepsilon_{2}^{-1}\right) P_{1}^{2}+P_{1} D_{d} D_{d}^{T} P_{1}+\varepsilon_{3} A_{c}^{T} A_{c}+\left(\varepsilon_{2}+\varepsilon_{4}\right) T_{1} \\
& \quad+\left(\varepsilon_{1}+\varepsilon_{10}\right) A_{d}^{T} A_{d}+\left(\varepsilon_{3}^{-1}+\varepsilon_{4}^{-1}+\varepsilon_{10}^{-1}\right) J^{T} P_{1}^{2} J+J^{T} P_{1} D D^{T} P_{1} J \\
& \quad+J^{T} P_{1} D_{d} D_{d}^{T} P_{1} J+\left(\varepsilon_{2}+\varepsilon_{4}\right) T_{2}+\delta_{1} I+R_{1}=0,
\end{aligned}
$$




$$
\begin{aligned}
P_{2} A & +A^{T} P_{2}+4 E^{T} E+P_{2} D D^{T} P_{2}+\left(\varepsilon_{5}^{-1}+\varepsilon_{6}^{-1}\right) P_{2}^{2}+P_{2} D_{d} D_{d}^{T} P_{2}^{T} \\
& +\varepsilon_{7} A^{T} A+\left(\varepsilon_{6}+\varepsilon_{8}\right) T_{1}+4 E_{d}^{T} E_{d}+\left(\varepsilon_{5}+\varepsilon_{9}\right) A_{d}^{T} A_{d} \\
& +\left(\varepsilon_{7}^{-1}+\varepsilon_{8}^{-1}+\varepsilon_{9}^{-1}\right) J^{T} P_{2}^{2} J+J^{T} P_{2} D D^{T} P_{2} J \\
& +J^{T} P_{2} D_{d} D_{d}^{T} P_{2} J+\left(\varepsilon_{6}+\varepsilon_{8}\right) T_{2}+\delta_{2} I+R_{2}=0 .
\end{aligned}
$$

Proof Consider the following candidate Lyapunov-Krasovskii functional:

$$
\begin{aligned}
V(t)= & (e(t)-J e(t-d))^{T} P_{1}(e(t)-J e(t-d))+\int_{t-d}^{t} e^{T}(s) Q_{1} e(s) d s \\
& +(x(t)-J x(t-d))^{T} P_{2}(x(t)-J x(t-d))+\int_{t-d}^{t} x^{T}(s) Q_{2} x(s) d s .
\end{aligned}
$$

Taking the time derivative of $V(t)$ for (33) yields

$$
\begin{aligned}
\dot{V}(t)= & 2(e(t)-J e(t-d))^{T} P_{1}\left[A_{c} e(t)+\Delta A(t) x(t)+\Delta A_{d}(t) x(t-d)+A_{d} e(t-d)+\Delta f\right] \\
& +e^{T}(t) Q_{1} e(t)-e^{T}(t-d) Q_{1} e(t-d)+x^{T}(t) Q_{2} x(t)-x^{T}(t-d) Q_{2} x(t-d) \\
& +2(x(t)-J x(t-d))^{T} P_{2}\left[(A+\Delta A(t)) x(t)+\left(A_{d}+\Delta A_{d}(t)\right) x(t-d)\right. \\
& +f(t, x(t), x(t-d))] \\
= & e^{T}(t)\left(P_{1} A_{c}+A_{c}^{T} P_{1}\right) e(t)+e^{T}(t) P_{1} \Delta A(t) x(t)+x^{T}(t) \Delta A^{T}(t) P_{1} e(t) \\
& +e^{T}(t) P_{1} \Delta A_{d}(t) x(t-d)+x^{T}(t-d) \Delta A_{d}^{T}(t) P_{1} e(t)+e^{T}(t) P_{1} A_{d} e(t-d) \\
& +e^{T}(t-d) A_{d}^{T} P_{1} e(t)+e^{T}(t) P_{1} \Delta f+\Delta f^{T} P_{1} e(t)-e^{T}(t-d) J^{T} P_{1} A_{c} e(t) \\
& -e^{T}(t) A_{c}^{T} P_{1} J e(t-d)-e^{T}(t-d) J^{T} P_{1} \Delta A(t) x(t)-x^{T}(t) \Delta A^{T}(t) P_{1} J e(t-d) \\
& -e^{T}(t-d) J^{T} P_{1} \Delta A_{d}(t) x(t-d) \\
& -x^{T}(t-d) \Delta A_{d}^{T}(t) P_{1} J e(t-d)-e^{T}(t-d) J^{T} P_{1} A_{d} e(t-d) \\
& -e^{T}(t-d) A_{d}^{T} P_{1} J e(t-d) \\
& +x^{T}(t) Q_{2} x(t)-e^{T}(t-d) J^{T} P_{1} \Delta f-\Delta f^{T} P_{1} J e(t-d)+e^{T}(t) Q_{1} e(t) \\
& -e^{T}(t-d) Q_{1} e(t-d) \\
& -x^{T}(t-d) Q_{2} x(t-d)+x^{T}(t)\left(P_{2} A+A^{T} P_{2}\right) x(t)+x^{T}(t) P_{2} \Delta A(t) x(t) \\
& +x^{T}(t) \Delta A(t)^{T} P_{2} x(t) \\
& +x^{T}(t) P_{2} A_{d} x(t-d)+x^{T}(t-d) A_{d}^{T} P_{2} x(t)+x^{T}(t) P_{2} \Delta A_{d} x(t-d) \\
& +x^{T}(t-d) \Delta A_{d}^{T} P_{2} x(t) \\
& +x^{T}(t) P_{2} f+f^{T} P_{2} x(t)-x^{T}(t-d) J^{T} P_{2} A x(t)-x^{T}(t) A^{T} P_{2} J x(t-d) \\
& -x^{T}(t-d) J^{T} P_{2} \Delta A x(t)-x^{T}(t) \Delta A^{T} P_{2} J x(t-d)-x^{T}(t-d) J^{T} P_{2} A_{d} x(t-d) \\
& -x^{T}(t-d) A_{d}^{T} P_{2} J x(t-d)-x^{T}(t-d) J^{T} P_{2} \Delta A_{d} x(t-d) \\
& -x^{T}(t-d) \Delta A_{d}^{T} P_{2} J x(t-d)-x^{T}(t-d) J^{T} P_{2} f-f^{T} P_{2} J x(t-d) . \\
& \\
&
\end{aligned}
$$


From Lemma 1 and Lemma 3, we have

$$
\begin{aligned}
& e^{T}(t) P_{1} \Delta A(t) x(t)+x^{T}(t) \Delta A^{T}(t) P_{1} e(t) \\
& =e^{T}(t) P_{1} D F E x(t)+x^{T}(t) E^{T} F^{T} D^{T} e(t) \leq e^{T}(t) P_{1} D D^{T} P_{1}^{T} e(t)+x^{T}(t) E^{T} E x(t), \\
& e^{T}(t) P_{1} \Delta A_{d}(t) x(t-d)+x^{T}(t-d) \Delta A_{d}^{T}(t) P_{1} e(t) \\
& \leq e^{T}(t) P_{1} D_{d} D_{d}^{T} P_{1}^{T} e(t)+x^{T}(t-d) E_{d}^{T} E_{d} x(t-d), \\
& -e^{T}(t-d) J^{T} P_{1} \Delta A(t) x(t)-x^{T}(t) \Delta A^{T}(t) P_{1} J e(t-d) \\
& \leq x^{T}(t) E^{T} E x(t)+e^{T}(t-d) J^{T} P_{1} D D^{T} P_{1} J e(t-d), \\
& -e^{T}(t-d) J^{T} P_{1} \Delta A_{d}(t) x(t-d)-x^{T}(t-d) \Delta A_{d}^{T}(t) P_{1} J e(t-d) \\
& \leq x^{T}(t-d) E_{d}^{T} E_{d} x(t-d)+e^{T}(t-d) J^{T} P_{1} D_{d} D_{d}^{T} P_{1} J e(t-d), \\
& e^{T}(t) P_{1} A_{d} e(t-d)+e^{T}(t-d) A_{d}^{T} P e(t) \leq \varepsilon_{1}^{-1} e^{T}(t) P_{1}^{2} e(t)+\varepsilon_{1} e^{T}(t-d) A_{d}^{T} A_{d} e(t-d), \\
& -e^{T}(t-d) J^{T} P_{1} A_{c} e(t)-e^{T}(t) A_{c}^{T} P_{1} J e(t-d) \\
& \leq \varepsilon_{3}^{-1} e^{T}(t-d) J^{T} P_{1}^{2} J e(t-d)+\varepsilon_{3} e^{T}(t) A_{c}^{T} A_{c} e(t), \\
& e^{T}(t) P_{1} \Delta f+\Delta f^{T} P_{1} e(t) \leq \varepsilon_{2}^{-1} e^{T}(t) P_{1}^{2} e(t)+\varepsilon_{2}\|\Delta f\|^{2}, \\
& -e^{T}(t-d) J^{T} P_{1} \Delta A(t) x(t)-x^{T}(t) \Delta A^{T}(t) P_{1} J e(t-d) \\
& \leq x^{T}(t) E^{T} E x(t)+e^{T}(t-d) J^{T} P_{1} D D^{T} P_{1} J e(t-d) \\
& -e^{T}(t-d) J^{T} P_{1} \Delta A_{d}(t) x(t-d)-x^{T}(t-d) \Delta A_{d}^{T}(t) P_{1} J e(t-d) \\
& \leq e^{T}(t-d) J^{T} P_{1} D_{d} D_{d}^{T} P_{1} J e(t-d)+x^{T}(t-d) E_{d}^{T} E_{d} x(t-d), \\
& -e^{T}(t-d) J^{T} P_{1} \Delta A(t) x(t)-x^{T}(t) \Delta A^{T}(t) P_{1} J e(t-d) \\
& \leq x^{T}(t) E^{T} E x(t)+e^{T}(t-d) J^{T} P_{1} D D^{T} P_{1} J e(t-d) \\
& -e^{T}(t-d) J^{T} P_{1} \Delta f-\Delta f^{T} P_{1} J e(t-d) \leq \varepsilon_{4}^{-1} e^{T}(t-d) J^{T} P_{1}^{2} J e(t-d)+\varepsilon_{4}\|\Delta f\|^{2}, \\
& x^{T}(t) P_{2} A_{d} x(t-d)+x^{T}(t-d) A_{d}^{T} P_{2} x(t) \leq \varepsilon_{5}^{-1} x^{T}(t) P_{2}^{2} x(t)+\varepsilon_{5} x^{T}(t-d) A_{d}^{T} A_{d} x(t-d), \\
& -e^{T}(t-d) J^{T} P_{1} \Delta A_{d}(t) x(t-d)-x^{T}(t-d) \Delta A_{d}^{T}(t) P_{1} J e(t-d) \\
& \leq e^{T}(t-d) J^{T} P_{1} D_{d} D_{d}^{T} P_{1} J e(t-d)+x^{T}(t-d) E_{d}^{T} E_{d} x(t-d), \\
& x^{T}(t) P_{2} \Delta A(t) x(t)+x^{T}(t) \Delta A(t)^{T} P_{2} x(t) \leq x^{T}(t) P_{2} D D^{T} P_{2} x(t)+x^{T}(t) E^{T} E x(t), \\
& x^{T}(t) P_{2} \Delta A_{d} x(t-d)+x^{T}(t-d) \Delta A_{d}^{T} P_{2} x(t) \\
& \leq x^{T}(t) P_{2} D_{d} D_{d}^{T} P_{2}^{T} x(t)+x^{T}(t-d) E_{d}^{T} E_{d} x(t-d), \\
& x^{T}(t) P_{2} f+f^{T} P_{2} x(t) \leq \varepsilon_{6}^{-1} x^{T}(t) P_{2}^{2} x(t)+\varepsilon_{6}\|f\|^{2}, \\
& -x^{T}(t-d) J^{T} P_{2} A x(t)-x^{T}(t) A^{T} P_{2} J x(t-d) \\
& \leq \varepsilon_{7}^{-1} x^{T}(t-d) J^{T} P_{2}^{2} J x(t-d)+\varepsilon_{7} x^{T}(t) A^{T}(t) A(t) x(t), \\
& -x^{T}(t-d) J^{T} P_{2} \Delta A(t) x(t)-x^{T}(t) \Delta A^{T}(t) P_{2} J x(t-d) \\
& \leq x^{T}(t) E^{T} E x(t)+x^{T}(t-d) J^{T} P_{2} D D^{T} P_{2} J x(t-d),
\end{aligned}
$$




$$
\begin{aligned}
& -x^{T}(t-d) J^{T} P_{2} \Delta A_{d} x(t-d)-x^{T}(t-d) \Delta A_{d}^{T} P_{2} J x(t-d) \\
& \quad \leq x^{T}(t-d) E_{d}^{T} E_{d} x(t-d)+x^{T}(t-d) J^{T} P_{2} D_{d} D_{d}^{T} P_{2} J x(t-d), \\
& -x^{T}(t-d) J^{T} P_{2} f-f^{T} P_{2} J x(t-d) \leq \varepsilon_{8}^{-1} x^{T}(t-d) J^{T} P_{2}^{2} J x(t-d)+\varepsilon_{8}\|f\|^{2} .
\end{aligned}
$$

Substituting (37)-(40) into (36), we have

$$
\begin{aligned}
& \dot{V}(t) \leq e^{T}(t)\left(P_{1} A_{c}+A_{c}^{T} P_{1}\right) e(t)+e^{T}(t) P_{1} D D^{T} P_{1}^{T} e(t)+x^{T}(t) E^{T} E x(t) \\
& +e^{T}(t) P_{1} D_{d} D_{d}^{T} P_{1}^{T} e(t) \\
& +x^{T}(t-d) E_{d}^{T} E_{d} x(t-d)+\varepsilon_{1}^{-1} e^{T}(t) P_{1}^{2} e(t)+\varepsilon_{1} e^{T}(t-d) A_{d}^{T} A_{d} e(t-d) \\
& +\varepsilon_{2}^{-1} e^{T}(t) P_{1}^{2} e(t) \\
& +\varepsilon_{2}\|\Delta f\|^{2}+\varepsilon_{3}^{-1} e^{T}(t-d) J^{T} P_{1}^{2} J e(t-d)+\varepsilon_{3} e^{T}(t) A_{c}^{T} A_{c} e(t) \\
& +e^{T}(t-d) J^{T} P_{1} D D^{T} P_{1} J e(t-d) \\
& +x^{T}(t) E^{T} E x(t)+e^{T}(t-d) J^{T} P_{1} D_{d} D_{d}^{T} P_{1} J e(t-d)+x^{T}(t-d) E_{d}^{T} E_{d} x(t-d) \\
& -e^{T}(t-d) J^{T} P_{1} A_{d} e(t-d)-e^{T}(t-d) A_{d}^{T} P_{1} J e(t-d) \\
& +\varepsilon_{4}^{-1} e^{T}(t-d) J^{T} P_{1}^{2} J e(t-d)+\varepsilon_{4}\|\Delta f\|^{2} \\
& +e^{T}(t) Q_{1} e(t)-e^{T}(t-d) Q_{1} e(t-d)+x^{T}(t) Q_{2} x(t)-x^{T}(t-d) Q_{2} x(t-d) \\
& +x^{T}(t)\left(P_{2} A+A^{T} P_{2}\right) x(t)+x^{T}(t) P_{2} D D^{T} P_{2} x(t)+x^{T}(t) E^{T} E x(t)+\varepsilon_{5}^{-1} x^{T}(t) P_{2}^{2} x(t) \\
& +\varepsilon_{5} x^{T}(t-d) A_{d}^{T} A_{d} x(t-d)+x^{T}(t) P_{2} D_{d} D_{d}^{T} P_{2}^{T} x(t)+x^{T}(t-d) E_{d}^{T} E_{d} x(t-d) \\
& +\varepsilon_{6}^{-1} x^{T}(t) P_{2}^{2} x(t)+\varepsilon_{6}\|f\|^{2}+\varepsilon_{7}^{-1} x^{T}(t-d) J^{T} P_{2}^{2} J x(t-d)+\varepsilon_{7} x^{T}(t) A^{T} A x(t) \\
& +x^{T}(t-d) J^{T} P_{2} D D^{T} P_{2} J x(t-d)+x^{T}(t) E^{T} E x(t)-x^{T}(t-d) J^{T} P_{2} A_{d} x(t-d) \\
& -x^{T}(t-d) A_{d}^{T} P_{2} J x(t-d)+x^{T}(t-d) J^{T} P_{2} D_{d} D_{d}^{T} P_{2} J x(t-d) \\
& +x^{T}(t-d) E_{d}^{T} E_{d} x(t-d)+\varepsilon_{8}^{-1} x^{T}(t-d) J^{T} P_{2}^{2} J x(t-d)+\varepsilon_{8}\|f\|^{2} \\
& =e^{T}(t)\left(P_{1} A_{c}+A_{c}^{T} P_{1}+P_{1} D D^{T} P_{1}+\left(\varepsilon_{1}^{-1}+\varepsilon_{2}^{-1}\right) P_{1}^{2}+P_{1} D_{d} D_{d}^{T} P_{1}+\varepsilon_{3} A_{c}^{T} A_{c}+Q_{1}\right) e(t) \\
& +x^{T}(t)\left[P_{2} A+A^{T} P_{2}+4 E^{T} E+Q_{2}+P_{2} D D^{T} P_{2}+\left(\varepsilon_{5}^{-1}+\varepsilon_{6}^{-1}\right) P_{2}^{2}\right. \\
& \left.+P_{2} D_{d} D_{d}^{T} P_{2}^{T}+\varepsilon_{7} A^{T} A\right] x(t) \\
& +x^{T}(t-d)\left[4 E_{d}^{T} E_{d}-Q_{2}+\varepsilon_{5} A_{d}^{T} A_{d}+\left(\varepsilon_{7}^{-1}+\varepsilon_{8}^{-1}\right) J^{T} P_{2}^{2} J\right. \\
& +J^{T} P_{2} D D^{T} P_{2} J-J^{T} P_{2} A_{d}-A_{d}^{T} P_{2} J \\
& \left.+J^{T} P_{2} D_{d} D_{d}^{T} P_{2} J\right] x(t-d)+\left(\varepsilon_{2}+\varepsilon_{4}\right)\|\Delta f\|^{2}+\left(\varepsilon_{6}+\varepsilon_{8}\right)\|f\|^{2}+e^{T}(t-d)\left[\varepsilon_{1} A_{d}^{T} A_{d}\right. \\
& +\left(\varepsilon_{3}^{-1}+\varepsilon_{4}^{-1}\right) J^{T} P_{1}^{2} J+J^{T} P_{1} D D^{T} P_{1} J+J^{T} P_{1} D_{d} D_{d}^{T} P_{1} J-J^{T} P_{1} A_{d} \\
& \left.-A_{d}^{T} P_{1} J-Q_{1}\right] e(t-d) \text {. }
\end{aligned}
$$

From Lemma 1 and (41), we get

$$
\begin{aligned}
\dot{V}(t) \leq & e^{T}(t)\left(P_{1} A_{c}+A_{c}^{T} P_{1}+P_{1} D D^{T} P_{1}+\left(\varepsilon_{1}^{-1}+\varepsilon_{2}^{-1}\right) P_{1}^{2}+P_{1} D_{d} D_{d}^{T} P_{1}+\varepsilon_{3} A_{c}^{T} A_{c}\right. \\
& \left.+Q_{1}+\left(\varepsilon_{2}+\varepsilon_{4}\right) T_{1}\right) e(t)
\end{aligned}
$$




$$
\begin{aligned}
& +x^{T}(t)\left[P_{2} A+A^{T} P_{2}+4 E^{T} E+Q_{2}\right. \\
& +P_{2} D D^{T} P_{2}+\left(\varepsilon_{5}^{-1}+\varepsilon_{6}^{-1}\right) P_{2}^{2}+P_{2} D_{d} D_{d}^{T} P_{2}^{T}+\varepsilon_{7} A^{T} A \\
& \left.+\left(\varepsilon_{6}+\varepsilon_{8}\right) T_{1}\right] x(t)+x^{T}(t-d)\left[4 E_{d}^{T} E_{d}-Q_{2}+\left(\varepsilon_{5}+\varepsilon_{9}\right) A_{d}^{T} A_{d}\right. \\
& +\left(\varepsilon_{7}^{-1}+\varepsilon_{8}^{-1}+\varepsilon_{9}^{-1}\right) J^{T} P_{2}^{2} J \\
& \left.+J^{T} P_{2} D D^{T} P_{2} J+J^{T} P_{2} D_{d} D_{d}^{T} P_{2} J+\left(\varepsilon_{6}+\varepsilon_{8}\right) T_{2}\right] x(t-d) \\
& +e^{T}(t-d)\left[\left(\varepsilon_{1}+\varepsilon_{10}\right) A_{d}^{T} A_{d}\right. \\
& +\left(\varepsilon_{3}^{-1}+\varepsilon_{4}^{-1}+\varepsilon_{10}^{-1}\right) J^{T} P_{1}^{2} J+J^{T} P_{1} D D^{T} P_{1} J+J^{T} P_{1} D_{d} D_{d}^{T} P_{1} J \\
& \left.+\left(\varepsilon_{2}+\varepsilon_{4}\right) T_{2}-Q_{1}\right] e(t-d) .
\end{aligned}
$$

Let

$$
\begin{aligned}
Q_{2}= & 4 E_{d}^{T} E_{d}+\left(\varepsilon_{5}+\varepsilon_{9}\right) A_{d}^{T} A_{d}+\left(\varepsilon_{7}^{-1}+\varepsilon_{8}^{-1}+\varepsilon_{9}^{-1}\right) J^{T} P_{2}^{2} J+J^{T} P_{2} D D^{T} P_{2} J \\
& +J^{T} P_{2} D_{d} D_{d}^{T} P_{2} J+\left(\varepsilon_{6}+\varepsilon_{8}\right) T_{2}+\delta_{2} I, \\
Q_{1}= & \left(\varepsilon_{1}+\varepsilon_{10}\right) A_{d}^{T} A_{d}+\left(\varepsilon_{3}^{-1}+\varepsilon_{4}^{-1}+\varepsilon_{10}^{-1}\right) J^{T} P_{1}^{2} J+J^{T} P_{1} D D^{T} P_{1} J \\
& +J^{T} P_{1} D_{d} D_{d}^{T} P_{1} J+\left(\varepsilon_{2}+\varepsilon_{4}\right) T_{2}+\delta_{1} I .
\end{aligned}
$$

Then we get

$$
\begin{aligned}
\dot{V}(t) \leq & e^{T}(t)\left(P_{1} A_{c}+A_{c}^{T} P_{1}+P_{1} D D^{T} P_{1}+\left(\varepsilon_{1}^{-1}+\varepsilon_{2}^{-1}\right) P_{1}^{2}+P_{1} D_{d} D_{d}^{T} P_{1}+\varepsilon_{3} A_{c}^{T} A_{c}+Q_{1}\right. \\
& \left.+\left(\varepsilon_{2}+\varepsilon_{4}\right) T_{1}\right) e(t)+x^{T}(t)\left[P_{2} A+A^{T} P_{2}+4 E^{T} E+Q_{2}+P_{2} D D^{T} P_{2}\right. \\
& \left.+\left(\varepsilon_{5}^{-1}+\varepsilon_{6}^{-1}\right) P_{2}^{2}+P_{2} D_{d} D_{d}^{T} P_{2}^{T}+\varepsilon_{7} A^{T} A+\left(\varepsilon_{6}+\varepsilon_{8}\right) T_{1}\right] x(t) \\
& -\delta_{2} x^{T}(t-d) x(t-d)-\delta_{1} e^{T}(t-d) e(t-d) .
\end{aligned}
$$

For simplicity, we denote

$$
\begin{aligned}
\Theta_{1}= & P_{1} A_{c}+A_{c}^{T} P_{1}+P_{1} D D^{T} P_{1}+\left(\varepsilon_{1}^{-1}+\varepsilon_{2}^{-1}\right) P_{1}^{2}+P_{1} D_{d} D_{d}^{T} P_{1} \\
& +\varepsilon_{3} A_{c}^{T} A_{c}+Q_{1}+\left(\varepsilon_{2}+\varepsilon_{4}\right) T_{1}, \\
\Theta_{2}= & P_{2} A+A^{T} P_{2}+4 E^{T} E+Q_{2}+P_{2} D D^{T} P_{2}+\left(\varepsilon_{5}^{-1}+\varepsilon_{6}^{-1}\right) P_{2}^{2}+P_{2} D_{d} D_{d}^{T} P_{2}^{T} \\
& +\varepsilon_{7} A^{T} A+\left(\varepsilon_{6}+\varepsilon_{8}\right) T_{1},
\end{aligned}
$$

where $Q_{1}$ and $Q_{2}$ are given by (42). Then (34), (35), and (44) indicate that

$$
\Theta_{1}=-R_{1}<0, \quad \Theta_{2}=-R_{2}<0 .
$$

Substituting (44) into (43) yields

$$
\begin{aligned}
\dot{V}(t) & \leq e^{T}(t) \Theta_{1} e(t)+x^{T}(t) \Theta_{2} x(t)-\delta_{2} x^{T}(t-d) x(t-d)-\delta_{1} e^{T}(t-d) e(t-d) \\
& \left.=\left[\begin{array}{c}
e(t) \\
x(t) \\
e(t-d) \\
x(t-d)
\end{array}\right]\right]^{T}\left[\begin{array}{cccc}
\Theta_{1} & 0 & 0 & 0 \\
0 & \Theta_{2} & 0 & 0 \\
0 & 0 & -\delta_{1} & 0 \\
0 & 0 & 0 & -\delta_{2}
\end{array}\right]\left[\begin{array}{c}
e(t) \\
x(t) \\
e(t-d) \\
x(t-d)
\end{array}\right]
\end{aligned}
$$




$$
\begin{aligned}
& \leq-\min \left(\lambda_{\min }\left(-\Theta_{1}\right), \lambda_{\min }\left(-\Theta_{2}\right), \delta_{1}, \delta_{2}\right)\left\|\left(e^{T}(t) \quad x^{T}(t) \quad e^{T}(t-d) \quad x^{T}(t-d)\right)\right\|^{2} \\
& \leq-\min \left(\lambda_{\min }\left(-\Theta_{1}\right), \lambda_{\min }\left(-\Theta_{2}\right), \delta_{1}, \delta_{2}\right)\left(\|e(t)\|^{2}+\|x(t)\|^{2}\right)<0,
\end{aligned}
$$

which implies that system (33) is asymptotically stable. This completes the proof of Theorem 3.

Theorem 4 For the given matrix $L$, system (33) is asymptotically stable if there exist positive scalars $\gamma_{i}(i=1,2,3,4)$ and positive definite matrices $P_{1}$ and $P_{2}$ such that the following LMIs hold:

$$
\begin{aligned}
& \left(\begin{array}{ccccccc}
\Gamma_{1} & P_{1} D & P_{1} & P_{1} D_{d} & J^{T} P_{1} & J^{T} P_{1} D & J^{T} P_{1} D_{d} \\
D^{T} P_{1} & -I & 0 & 0 & 0 & 0 & 0 \\
P_{1} & 0 & -\gamma_{1} I & 0 & 0 & 0 & 0 \\
D_{d}^{T} P_{1} & 0 & 0 & -I & 0 & 0 & 0 \\
P_{1} & 0 & 0 & 0 & -\gamma_{2} I & 0 & 0 \\
D^{T} P_{1} J & 0 & 0 & 0 & 0 & -I & 0 \\
D_{d}^{T} P_{1} J & 0 & 0 & 0 & 0 & 0 & -I
\end{array}\right)<0, \\
& \left(\begin{array}{ccccccc}
\Gamma_{2} & P_{2} D & P_{2} & P_{2} D_{d} & J^{T} P_{2} & J^{T} P_{2} D & J^{T} P_{2} D_{d} \\
D^{T} P_{2} & -I & 0 & 0 & 0 & 0 & 0 \\
P_{1} & 0 & -\gamma_{3} I & 0 & 0 & 0 & 0 \\
D_{d}^{T} P_{2} & 0 & 0 & -I & 0 & 0 & 0 \\
P_{2} J & 0 & 0 & 0 & -\gamma_{4} I & 0 & 0 \\
D^{T} P_{2} J & 0 & 0 & 0 & 0 & -I & 0 \\
D_{d}^{T} P_{2} J & 0 & 0 & 0 & 0 & 0 & -I
\end{array}\right)<0,
\end{aligned}
$$

where

$$
\begin{aligned}
& \Gamma_{1}=P_{1} A_{c}+A_{c}^{T} P_{1}+3 \gamma_{2} A_{c}^{T} A_{c}+\left(2 \gamma_{1}+3 \gamma_{2}\right)\left(T_{1}+T_{2}\right)+\left(2 \gamma_{1}+3 \gamma_{2}\right) A_{d}^{T} A_{d}, \\
& \Gamma_{2}=P_{2} A+A^{T} P_{2}+4 E^{T} E+3 \gamma_{4} A^{T} A+\left(2 \gamma_{3}+3 \gamma_{4}\right)\left(T_{1}+T_{2}+A_{d}^{T} A_{d}\right)+4 E_{d}^{T} E_{d} .
\end{aligned}
$$

Proof Consider the following candidate Lyapunov-Krasovskii functional:

$$
\begin{aligned}
V(t)= & (e(t)-J e(t-d))^{T} P_{1}(e(t)-J e(t-d))+\int_{t-d}^{t} e^{T}(s) Q_{1} e(s) d s \\
& +(x(t)-J x(t-d))^{T} P_{2}(x(t)-J x(t-d))+\int_{t-d}^{t} x^{T}(s) Q_{2} x(s) d s .
\end{aligned}
$$

Time derivative of $V(t)$ along the trajectory of (33) yields

$$
\begin{aligned}
\dot{V}(t) \leq & e^{T}(t)\left(P_{1} A_{c}+A_{c}^{T} P_{1}+P_{1} D D^{T} P_{1}+\left(\varepsilon_{1}^{-1}+\varepsilon_{2}^{-1}\right) P_{1}^{2}+P_{1} D_{d} D_{d}^{T} P_{1}+\varepsilon_{3} A_{c}^{T} A_{c}+Q_{1}\right. \\
& \left.+\left(\varepsilon_{2}+\varepsilon_{4}\right) T_{1}\right) e(t)+x^{T}(t)\left[P_{2} A+A^{T} P_{2}+4 E^{T} E+Q_{2}+P_{2} D D^{T} P_{2}\right. \\
& \left.+\left(\varepsilon_{5}^{-1}+\varepsilon_{6}^{-1}\right) P_{2}^{2}+P_{2} D_{d} D_{d}^{T} P_{2}^{T}+\varepsilon_{7} A^{T} A+\left(\varepsilon_{6}+\varepsilon_{8}\right) T_{1}\right] x(t) \\
& +x^{T}(t-d)\left[4 E_{d}^{T} E_{d}-Q_{2}+\left(\varepsilon_{5}+\varepsilon_{9}\right) A_{d}^{T} A_{d}\right. \\
& \left.+\left(\varepsilon_{7}^{-1}+\varepsilon_{8}^{-1}+\varepsilon_{9}^{-1}\right) J^{T} P_{2}^{2} J+J^{T} P_{2} D D^{T} P_{2} J+J^{T} P_{2} D_{d} D_{d}^{T} P_{2} J+\left(\varepsilon_{6}+\varepsilon_{8}\right) T_{2}\right] x(t-d)
\end{aligned}
$$




$$
\begin{aligned}
& +e^{T}(t-d)\left[\left(\varepsilon_{1}+\varepsilon_{10}\right) A_{d}^{T} A_{d}+\left(\varepsilon_{3}^{-1}+\varepsilon_{4}^{-1}+\varepsilon_{10}^{-1}\right) J^{T} P_{1}^{2} J+J^{T} P_{1} D D^{T} P_{1} J\right. \\
& \left.+J^{T} P_{1} D_{d} D_{d}^{T} P_{1} J+\left(\varepsilon_{2}+\varepsilon_{4}\right) T_{2}-Q_{1}\right] e(t-d) .
\end{aligned}
$$

Let

$$
\begin{aligned}
Q_{2}= & 4 E_{d}^{T} E_{d}+\left(\varepsilon_{5}+\varepsilon_{9}\right) A_{d}^{T} A_{d}+\left(\varepsilon_{7}^{-1}+\varepsilon_{8}^{-1}+\varepsilon_{9}^{-1}\right) J^{T} P_{2}^{2} J+J^{T} P_{2} D D^{T} P_{2} J \\
& +J^{T} P_{2} D_{d} D_{d}^{T} P_{2} J+\left(\varepsilon_{6}+\varepsilon_{8}\right) T_{2}, \\
Q_{1}= & \left(\varepsilon_{1}+\varepsilon_{10}\right) A_{d}^{T} A_{d}+\left(\varepsilon_{3}^{-1}+\varepsilon_{4}^{-1}+\varepsilon_{10}^{-1}\right) J^{T} P_{1}^{2} J+J^{T} P_{1} D D^{T} P_{1} J \\
& +J^{T} P_{1} D_{d} D_{d}^{T} P_{1} J+\left(\varepsilon_{2}+\varepsilon_{4}\right) T_{2} .
\end{aligned}
$$

Substituting (47) into (46) yields

$$
\begin{aligned}
\dot{V}(t) \leq & e^{T}(t)\left(P_{1} A_{c}+A_{c}^{T} P_{1}+P_{1} D D^{T} P_{1}+\left(\varepsilon_{1}^{-1}+\varepsilon_{2}^{-1}\right) P_{1}^{2}+P_{1} D_{d} D_{d}^{T} P_{1}+\varepsilon_{3} A_{c}^{T} A_{c}+Q_{1}\right. \\
& \left.+\left(\varepsilon_{2}+\varepsilon_{4}\right) T_{1}\right) e(t)+x^{T}(t)\left[P_{2} A+A^{T} P_{2}+4 E^{T} E+Q_{2}+P_{2} D D^{T} P_{2}\right. \\
& \left.\left.+\left(\varepsilon_{5}^{-1}+\varepsilon_{6}^{-1}\right) P_{2}^{2}+P_{2} D_{d} D_{d}^{T} P_{2}^{T}+\varepsilon_{7} A^{T} A+\left(\varepsilon_{6}+\varepsilon_{8}\right) T_{1}\right] x(t)\right) .
\end{aligned}
$$

Let $\varepsilon_{1}=\varepsilon_{2}=2 \gamma_{1}, \varepsilon_{3}=\varepsilon_{4}=\varepsilon_{10}=3 \gamma_{2}, \varepsilon_{5}=\varepsilon_{6}=2 \gamma_{3}, \varepsilon_{7}=\varepsilon_{8}=\varepsilon_{9}=3 \gamma_{4}$. From Lemma 2, (45) implies that $\dot{V}<0$, which implies that system (33) is asymptotically stable. This completes the proof of Theorem 4 .

Remark 2 It is well known that if system (33) is asymptotically stable, then both systems (32) and (27) are asymptotically stable.

\section{Numerical example}

In this section, we demonstrate the theory developed in this paper by means of a simple example.

Consider nonlinear neutral delay system (20)-(21) with

$$
\begin{aligned}
& A=\left(\begin{array}{cc}
-3 & -1 \\
1 & -8
\end{array}\right), \quad A_{1}=\left(\begin{array}{cc}
3 & 1 \\
4 & 2+\sqrt{2}
\end{array}\right), \quad C=\left(\begin{array}{ll}
1 & -2
\end{array}\right), \quad C_{1}=\left(\begin{array}{ll}
2 & 1
\end{array}\right), \\
& J=0.11, \quad f(t, x(t), x(t-1))=\left(\begin{array}{c}
\frac{1}{2} \cos t x_{1}(t)+\frac{3}{4} \sin t x_{2}(t-1) \\
\frac{1}{2} \sin t x_{2}(t)
\end{array}\right) .
\end{aligned}
$$

It is easy to obtain that

$$
\begin{aligned}
& f(t, x(t), x(t-1))-f(t, \hat{x}(t), \hat{x}(t-1)) \\
& \quad=\left(\begin{array}{c}
\frac{1}{2} \cos t\left(x_{1}(t)-\hat{x}_{1}(t)\right)+\frac{3}{4} \sin t\left(x_{2}(t-1)-\hat{x}_{2}(t-1)\right) \\
\frac{1}{2} \sin t\left(x_{2}(t)-\hat{x}_{2}(t)\right)
\end{array}\right) .
\end{aligned}
$$

So,

$$
\|f(t, x(t), x(t-1))-f(t, \hat{x}(t), \hat{x}(t-1))\|^{2} \leq \frac{5}{8}\|x(t)-\hat{x}(t)\|^{2}+\frac{9}{16}\|x(t-1)-\hat{x}(t-1)\|^{2},
$$


i.e., $T_{1}=\frac{5}{8} I, T_{2}=\frac{9}{16} I$. Let $L=\left(\begin{array}{l}1 \\ 2\end{array}\right)$, then

$$
\begin{aligned}
& A_{c}=A-L C=\left(\begin{array}{cc}
-3 & -1 \\
1 & -8
\end{array}\right)-\left(\begin{array}{l}
1 \\
2
\end{array}\right)\left(\begin{array}{ll}
1 & -2
\end{array}\right)=\left(\begin{array}{cc}
-4 & 1 \\
-1 & -4
\end{array}\right), \\
& A_{d}=A_{1}-L C_{1}=\left(\begin{array}{cc}
3 & 1 \\
4 & 2+\sqrt{2}
\end{array}\right)-\left(\begin{array}{l}
1 \\
2
\end{array}\right)\left(\begin{array}{ll}
2 & 1
\end{array}\right)=\left(\begin{array}{cc}
1 & 0 \\
0 & \sqrt{2}
\end{array}\right) .
\end{aligned}
$$

Taking $\varepsilon_{1}=\varepsilon_{4}=10, \varepsilon_{2}=\varepsilon_{3}=\varepsilon_{5}=1$, one can easily verify that linear matrix inequality (26) has a solution

$$
P=\left(\begin{array}{cc}
20 & 0 \\
0 & 20
\end{array}\right)
$$

According to Corollary 2, system (24) is asymptotically stable.

\section{Conclusion}

In this paper, the problem of observer design for a class of nonlinear neutral systems with time delay is discussed. Firstly, we present some sufficient conditions for the existence of observers of a class of nonlinear neutral systems with time-varying delay. An effective algebraic matrix equation approach is developed. Then a design method of the observer, which is dependent on the solution of the linear matrix inequality, is proposed. Furthermore, we consider robust observer designs for a class of nonlinear neutral systems with time delay and uncertainties. The sufficient conditions which guarantee that the observer error converges asymptotically to zero are given. Finally, a numerical example is provided to show the applicability of the developed results.

\section{Competing interests}

The authors declare that they have no competing interests.

Authors' contributions

Each of the authors read and approved the final version of the manuscript.

\section{Author details}

'School of Science, Tianjin Polytechnic University, Tianjin, 300387, China. ${ }^{2}$ Department of Environmental Economics, Tianjin Polytechnic University, Tianjin, 300387, China.

\section{Acknowledgements}

This work is supported by the Natural Science Foundation of Tianjin under Grant 11JCYBJC06800, the National Social Science Key Foundation of China under Grant 13AZD011, and the National Social Science Foundation of China under Grant 12BJY025.

Received: 3 December 2013 Accepted: 2 April 2014 Published: 07 May 2014

\section{References}

1. Yia, S, Ulsoya, AG, Nelsonb, PW: Design of observer-based feedback control for time-delay systems with application to automotive power train control. J. Franklin Inst. 347, 358-376 (2010)

2. Wang, Y, Lynch, AF: Observer design using a generalized time-scaled block triangular observer form. Syst. Control Lett. 58, 346-352 (2009)

3. Frank, PM: Fault diagnosis in dynamic systems using analytical and knowledge-based redundancy: a survey and some new results. Automatica 26, 459-474 (1990)

4. Marquez, HJ, Riaz, M: Robust state observer design with application to an industrial boiler system. Control Eng. Pract. 13(6), 713-728 (2005)

5. Chen, M, Chen, C: Robust nonlinear observer for Lipschitz nonlinear systems subject to disturbances. IEEE Trans. Autom. Control 52(12), 2365-2369 (2007)

6. Barmish, BR, Galimidi, AR: Robustness of Luenberger observers: linear systems stabilized via nonlinear control. Automatica 22, 413-423 (1986) 
7. Dong, $Y$, Wang, $H$, Wang, $Y$ : Design of observers for nonlinear systems with $\mathrm{H}_{\infty}$ performance analysis. Math. Methods Appl. Sci. 37, 718-725 (2014)

8. Dong, Y, Zhang, Y, Zhang, X: Design of observer-based feedback control for a class of discrete-time nonlinear systems with time-delay. Appl. Comput. Math. 13(1), 107-121 (2014)

9. Kharitonov, V, Mondie, S, Collado, J: Exponential estimates for neutral time-delay systems: an LMI approach. IEEE Trans. Autom. Control 50(5), 666-670 (2005)

10. $\mathrm{Wu}, \mathrm{M}, \mathrm{He}, \mathrm{Y}, \mathrm{She}, \mathrm{JH}$ : New delay-dependent stability criteria and stabilizing method for neutral systems. IEEE Trans. Autom. Control 49(12), 2266-2271 (2004)

11. Shatyrko, A, Diblik, J, Khusainov, D, Ruzickova, M: Stabilization of Lur'e-type nonlinear control systems by Lyapunov-Krasovskii functionals. Adv. Differ. Equ. 2012, 229 (2012). doi:10.1186/1687-1847-2012-229

12. Trinh, H, Aldeen, M: A memoryless state observer for discrete time-delay systems. IEEE Trans. Autom. Control 42 1572-1577 (1997)

13. Yao, YX, Zhang, YM, Kovacevic, R: Functional observer and state feedback for input time-delay systems. Int. J. Control 66, 603-617 (1997)

14. Lien, CH, Yu, KW, Lin, YF, Chung, YJ, Chung, LY: Exponential convergence rate estimation for uncertain delayed neural networks of neutral type. Chaos Solitons Fractals 40, 2491-2499 (2009)

15. Yu, KW, Lien, CH: Stability criteria for uncertain neutral systems with interval time-varying delays. Chaos Solitons Fractals 38, 650-657 (2008)

16. Shatyrko, AV, Khusainov, DY: Absolute interval stability of indirect regulating systems of neutral type. J. Autom. Inf. Sci. 42, 43-54 (2010)

17. Shatyrko, AV, Khusainov, DY, Diblik, J, Bastinec, A, Rivolova, J: Estimates of perturbation of nonlinear indirect interval control system of neutral type. J. Autom. Inf. Sci. 43, 13-28 (2011)

18. Shatyrko, AV, Khusainov, DY: Investigation of absolute stability of nonlinear systems of special kind with aftereffect by Lyapunov functions method. J. Autom. Inf. Sci. 43, 61-75 (2011)

19. Dong, Y, Mei, S: State observers for a class of multi-output nonlinear dynamic systems. Nonlinear Anal., Theory Methods Appl. 74(14), 4738-4745 (2011)

20. Krstic, M, Kanellakopoulos, I, Kokotovic, PV: Nonlinear and Adaptive Control Design. Wiley, New York (1995)

21. Rajamani, R: Observers for Lipschitz nonlinear systems. IEEE Trans. Autom. Control 43, 397-401 (1998)

22. Kreisselmeier, G, Engel, R: Nonlinear observers for autonomous Lipschitz continuous systems. IEEE Trans. Autom. Control 48, 451-464 (2003)

23. Dong, Y, Liu, J, Mei, S: Observer design for a class of nonlinear discrete-time systems with time-delay. Kybernetika 49 342-359 (2013)

24. Dong, Y, Yang, Y: Observer design for a class of multi-input multi-output nonlinear systems. Int. J. Syst. Sci. 42(4) 695-703 (2011)

25. Wang, Z, Lam, JK, Burnham, J: Stability analysis and observer design for neutral delay systems. IEEE Trans. Autom. Control 47, 478-482 (2002)

10.1186/1687-1847-2014-133

Cite this article as: Dong et al.: Stability analysis of nonlinear observer for neutral uncertain time-delay systems. Advances in Difference Equations 2014, 2014:133

\section{Submit your manuscript to a SpringerOpen ${ }^{\circ}$ journal and benefit from:}

- Convenient online submission

Rigorous peer review

- Immediate publication on acceptance

- Open access: articles freely available online

- High visibility within the field

- Retaining the copyright to your article 